1. I wish to thank B. C. Kent and K. A Spriggel, of the Goodyear Research laboratory, for their valuable assistance in preparing and testing the hardware and software for the debug system. Appreciation is also expressed to the
Goodyear Tire \& Rubber Company and the Research Division for their support of this project and permission to publicize the result.

\title{
A spectral analysis program for the processing of neuro-electric data
}

FRANCESCA URBANO, STEPHEN G. PAUKER, and FRANK R. ERVIN, STANLEY COBB LABORATORIES FOR PSYCHIATRIC RESEARCH, MASSACHUSETTS GENERAL HOSPITAL, Boston, Massachusetts 02114

The Spectral Analysis Program is designed to perform the following kinds of spectral analysis, using the Cooley-Tukey Fast Fourier Transform Algorithm: cross correlation, cross power spectrum, Fourier transform, inverse Fourier transform, and double Fourier transform (the process of transforming two real functions simultaneously). These operations are performed on two blocks of core that can be filled through time-sequential sampling of an external analog signal or from previously sampled data that have been stored on DECtape. Through the use of nine Teletype commands, the user can manipulate input and output and select a particular type of spectral analysis. Output may be displayed on a $30 D$ scope stored on DECtape or turned into hard copy by a Calcomp plotter used for processing neuroelectric data at the Stanley Cobb Laboratories for Psychiatric Research.

Equipped $^{1}$ with an 9K PDP-7 computer, two DECtape units, and some standard input-output devices, we set about the task of providing neurophysiologists at the Stanley Cobb Laboratories for Psychiatric Research with a flexible tool for the analysis of human brain-wave activity. The rationale for our approach to this problem, the Spectral Analysis Program that was the solution, and the applications that it made possible are the topics to which this paper is devoted.

\section{BACKGROUND}

The traditional measure of brain-wave activity is the electroencephalogram, or EEG, which consists of a time-varying voltage measured between two points on the skull or in the brain (see Fig. 1). There are many factors that influence the waveform of an EEG. Two such factors are: (1) location of the probe on the skull and $(2)$ the physiological condition of the test patient-asleep vs awake, anxious vs calm, tired vs alert. In broad terms, the prime objective of the investigator in studying the EEG is to detect patterns or changes in the waveform that correspond to behavioral state. For example, in the ideal situation, the researcher would like to find that a specific EEG was characteristic of the schizophrenic but did not occur in the nonschizophrenic. He might then be able to diagnose schizophrenia as well as determine how his patient was responding to treatment. The soundness of such an approach is based on the assumption that "brain wave phenomena are intimately involved with the state of the organism and are not just irrelevant noise [Hanley, Walter, Rhodes, \& Adey, 1969]."

\section{METHODS OF EEG ANALYSIS}

The era preceding large, high-speed digital computers found EEG analysis in a qualitative state. Three basic techniques, all visual in nature, were available to the investigator:

(1) He could look for a dominant frequency in the waveform. Motivated by the-impression that the EEG was an inherently oscillatory phenomenon, the investigator found some important clues as to brain function. A high frequency $E E G$ was found to correlate with a hyperalert, anxious state, while a low-frequency wave was indicative of a drowsy, calm state.

(2) The detection of symmetry in EEG recorded from physiologically symmetric points in the brain was another visual technique with limited usefulness. In the normal brain, recording from two identical structures yields two identical waveforms. Asymmetry has led neurophysiologists to suspect malfunction or obstruction in one of the hemispheres. Indeed, brain tumors and blood clots have been found in these cases. But the capacity for refinement of this clinical tool is unlimited. The pressing need is for more sophisticated quantitative analysis techniques.

(3) The visual detection of unique patterns in EEG has produced three major results: (a) The discovery of the $3 / \mathrm{sec}$ spike and waveform characteristic of petit mal epilepsy. (b) The separation of sleep into five stages, distinguishable both by the pattern of their EEG and other physical signs. (c) The discovery of the alpha wave and blocking phenomena; this pattern is a sinusoidal 8- to 12-cps wave, recorded from the occipital region of the head and present in the awake, relaxed patient. Its most striking feature is its disappearance, or "blocking," upon presentation of a stimulus.

One cannot ignore the importance of the above discoveries. Yet, like the former methods of visual inspection, visual pattern recognition (3) has had limited success as it completely precludes the possibility of other patterns hidden in noise.

The ability of the present-day computer to perform complicated mathematical analysis on large masses of data has revolutionized EEG analysis and given investigators a powerful quantitative tool.

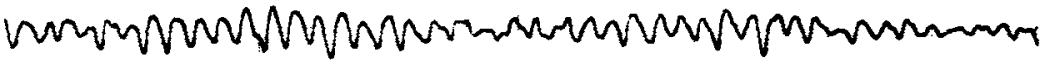

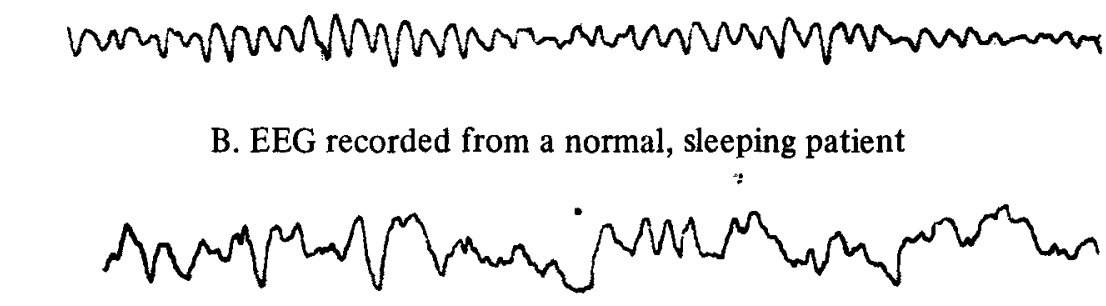

A. EEG recorded from a normal, awake relaxed patient

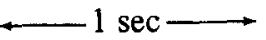




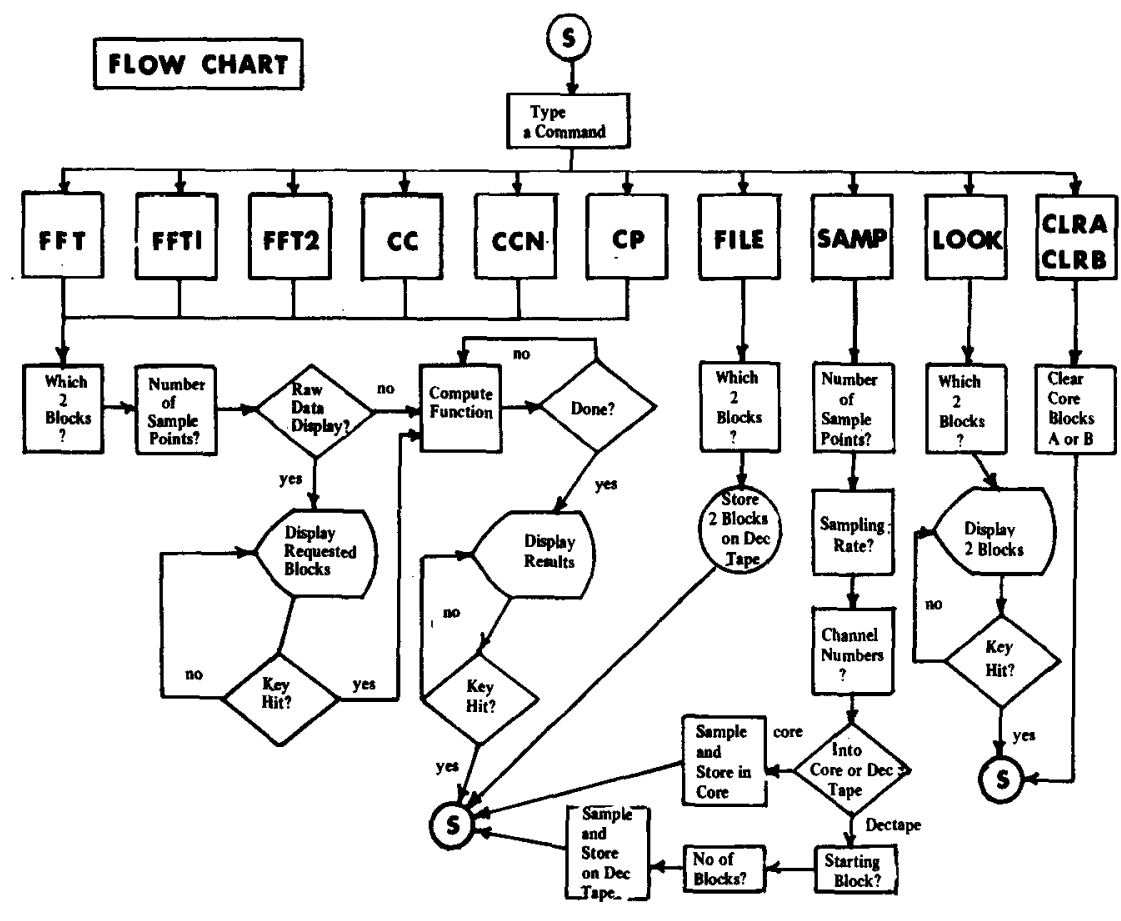

\begin{tabular}{|c|c|c|c|c|c|}
\hline \multirow[b]{2}{*}{ FUNCTION } & \multirow[b]{2}{*}{ COMnND } & \multirow[b]{2}{*}{ DESCRIPTION OF $\mathrm{Cl}$} & \multicolumn{3}{|c|}{ EXECUTION TIME, $N=$} \\
\hline & & & 1024 & 512 & $\underline{256}$ \\
\hline Fourler Transform & FFT & $\begin{array}{l}\text { Calculates the Pourler transform } 1 \\
\text { of one complex function. The } \\
\text { function's real and imginary } \\
\text { parts are of length } N \text {, wich } \\
\text { must be pover of } 2 \text { less than } \\
\text { or equel to } 1024 \text {. }\end{array}$ & 16 & $\begin{array}{l}7 \\
\text { sec. }\end{array}$ & $\begin{array}{c}4 \\
\text { soc. }\end{array}$ \\
\hline $\begin{array}{l}\text { Inverse Fourier } \\
\text { Transform }\end{array}$ & FFTI & $\begin{array}{l}\text { Finds the inverse transform of } \\
\text { a single complex function. The } \\
\text { runetion's real and Imaginary } \\
\text { pert's are both of length N, } \\
\text { which is restrieted as above. }\end{array}$ & 17 & 8 & 4 \\
\hline $\begin{array}{l}\text { Double Fourler } \\
\text { Transform }\end{array}$ & FFT2 & $\begin{array}{l}\text { Celculates two transforms simul- } \\
\text { taneously. Both functions are } \\
\text { resl, and of length N, restricted } \\
\text { as above. }\end{array}$ & 18 & 9 & 5 \\
\hline Cross Correlation & $\mathrm{CC}$ & $\begin{array}{l}\text { Calculates eross correlation } \\
\text { runction. Both input functions } \\
\text { must be real, and of equal length } \\
N \text {, where } N \text { is restricted as above }\end{array}$ & $\begin{array}{l}32 \\
\text { hi, }\end{array}$ & 15 & 7 \\
\hline $\begin{array}{l}\text { Normelized Cross } \\
\text { Correlation }\end{array}$ & $\mathrm{CCN}$ & $\begin{array}{l}\text { Calculates. a cross correlation } \\
\text { runction and normalizes it by } \\
\text { dividing each velue by SoRr( } \\
\text { SUM(F1)(F1))(SLM(F2)(F2))). The } \\
\text { input functlons are of unequal } \\
\text { length, the longer boing of } \\
\text { length } N \text {, the shorter, of longth } \\
\text { M. }\end{array}$ & 31 & 15 & 6 \\
\hline $\begin{array}{l}\text { Cross Power } \\
\text { Spectrum }\end{array}$ & CP & $\begin{array}{l}\text { Celculates a cross power spectrum } \\
\text { runction. Both input runetions } \\
\text { must be real, and of equal lengtl. } \\
\mathrm{N} \text {, whore } \mathrm{N} \text { is as ebove. }\end{array}$ & 28 & 13 & 6 \\
\hline
\end{tabular}

Fig. 2. Flow chart.

Included among the rigorous techniques made available by the new computer flexibility are: (1) cross-correlation functions and (2) power spectral density functions. The computations of these functions constitute the main portion of the Spectral Analysis Program.

\section{UNIQUE ADVANTAGES OF A SMALL COMPUTER FOR EEG ANALYSIS}

The small computer is extremely well suited for the processing of neuroelectric data. The reasons for this are twofold:

(1) The E can interact effectively with the machine. He can make the decision to alter the course of data processing after it has already begun. He can decide on the basis of results from immediate analysis to treat his data differently. Perhaps most significant is that the $\mathrm{E}$ can more easily make use of peripheral equipment to examine raw data before they are processed. This allows him to verify visually certain fundamental assumptions on the nature of his data, such as stationarity, randomness, and normality. EEG data will not always satisfy these requirements and a skilled $E$ is often needed to determine how far the rigor of these techniques is being stretched.

(2) The machine may be used in an on-line situation where it can make decisions as to the course of the experiment. For example, in a situation where a response to a stimulus is being investigated by examining the EEG, the validity of the record as a measure of the S's response is greater when the S's EEG has been stabilized (in other words, when his EEG exhibits "stationarity"). The computer can determine when stationarity exists either from a set of arbitrary criteria imposed by the $\mathrm{E}$ or by performing statistical tests. It will then present the stimulus accordingly. This has been shown in our laboratory to be a very useful technique.

\section{THE SPECTRAL ANALYSIS PROGRAM}

The Spectral Analysis Program, created to analyze EEG data on a PDP-7, has small-machine flexibility and an interactive user-oriented language, and it employs the Cooley-Tukey algorithm to speed up computation of the Fourier transform. The program computes correlation functions and power spectral density functions. It includes calculation of autocorrelation, cross correlation, power spectra, cross power spectra, and direct and inverse Fourier transforms. These operations are

Fig. 3. Table of processing commands. 
Fig. 4. A typical computer-user dialogue.

performed on two 1024-word blocks of core that can be filled through time-sequential sampling of an external analog signal or from previously sampled data that have been stored on DECtape. In the case of our EEG analysis, the data are collected on seven-channel magnetic tape and placed back on a tape recorder in to the analog-to-digital converter at process time. Through the use of nine Teletype commands, the user can manipulate input and output and select a particular type of spectral analysis. Output may be displayed on a 30D scope, stored on DECtape, or turned into hard copy on a Calcomp plotter. The program is organized to execute one command at a time and then request another one (see flow chart in Fig. 2). This provides the user with maximum control over his options.

\section{Processing Commands}

The six Teletype commands that perform correlation and spectral analysis are described in detail in Fig. 3. The maximum execution time for the longest of these analyses, the cross-correlation of 1024 points, is $30 \mathrm{sec}$. The execution time of each processing command is a function of $\mathbf{N}$, the number of sample points taken. It may be as short as $4 \mathrm{sec}$ for 256 points. In this program, command execution time was speeded up by as much as $99 \%$ by the implementation of the Cooley-Tukey Fast Fourier Transform Algorithm.

\section{Input-Output Commands}

There are three basic input-output commands, "FILE," "SAMP," and "LOOK," and two commands, "CLRA" and "CLRB," which create zero buffers in core. The file command ("FILE") allows the user to store two blocks of input data or processed data on DECtape. The sample command ("SAMP") enables him to sample an external analog signal at a desired rate and for a desired length of time. The data may be sampled two blocks at a time and fed into core, or it may be collected continuously in large quantities and dumped on DECtape. The display command ("LOOK") will generate a scope display of any two blocks on DECtape or of the two data buffers in core. Subsequent plotting of the displayed data will take place when the user sets an accumulator switch.

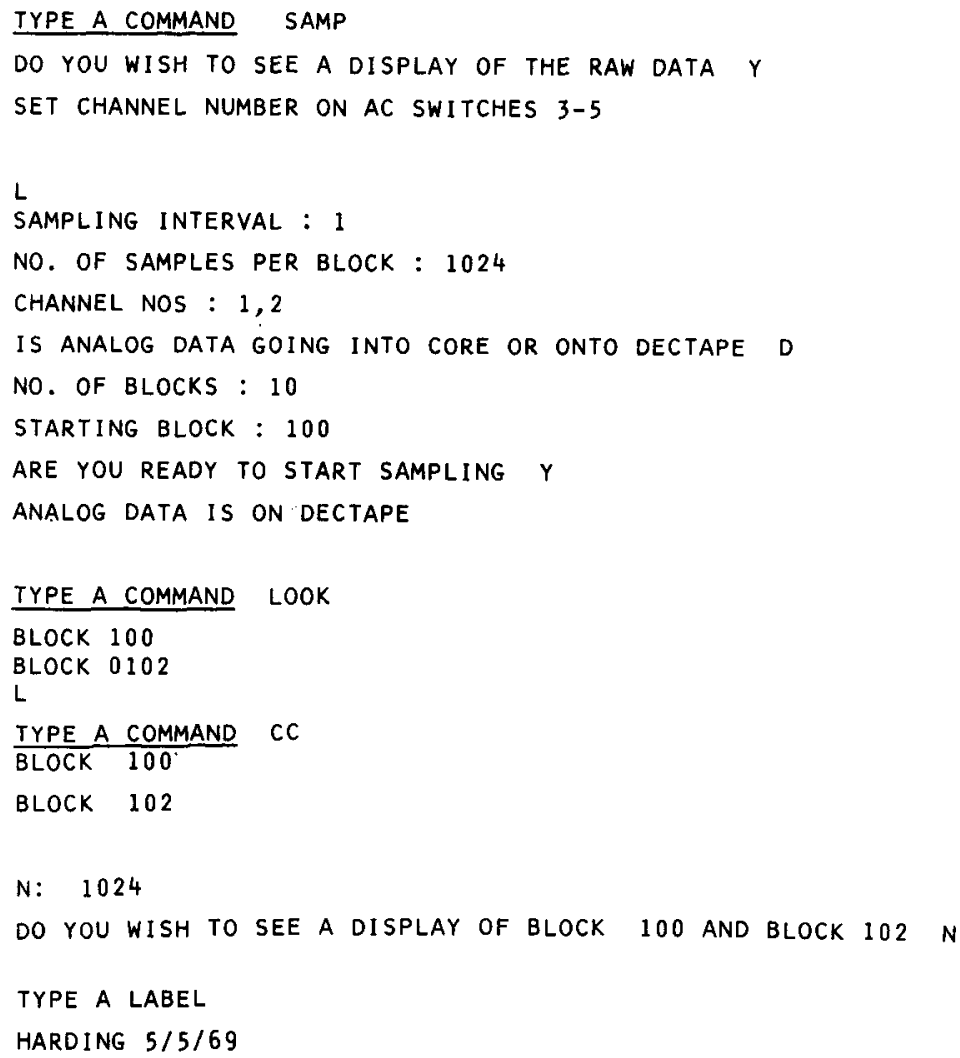

A typical sequence of commands for the processing of an EEG record might proceed as is documented by the computer-user "conversation" in Fig. 4.

\section{APPLICATIONS}

The functions made available by the Spectral Analysis Program have a wide range of applications for the neurophysiologist. The cross-correlation functions allow him to examine data in the time domain, while power spectral density functions show how they are distributed in the frequency domain. Both analyses aim towards reducing the signal-to-noise ratio. Both the autocorrelation and the power spectral density function will detect hidden periodicities in a waveform, if they exist. Cross correlation is especially well suited to prediction and filter theory. If the investigator is searching for a particular waveshape that he suspects is obliterated by noise, he may create a template of the predicted pattern he seeks and cross correlate it with his noisy data. The location of epileptic centers in the brain may be found with cross-correlation techniques by measuring time delays between spikes. This is similar to the technique that locates an earthquake or a nuclear explosion from miles away. Now when our neurophysiologists ask, "Can I discover anything about the state of the organism or the relationship between different regions of the brain by examining the EEG?" they have a sensitive quantitative tool with which to approach their questions.

\section{REFERENCE}

HANLEY, J., WALTER, D. O., RHODES, J. M., \& ADEY, W. R. Chimpanzee performance: Computer analysis of electroencephalograms. Mental Health Digest, 1969, 1, 44.

\section{Note}

1. The hardware used by the Spectral Analysis Program includes the following: Digital Equipment Corporation's PDP-7 Computer, CRT Oscilloscope Type 30D, A-D Converter Type 138, DEC tape Transport and Control Types 555 and 550, and High Speed Paper Tape Reader and Punch; Calcomp's Digital Plotter Type 565; and a KSF 33 Teletype. 